\title{
An update on stem cell therapy for neurological disorders: cell death pathways as therapeutic targets
}

\author{
Sydney Corey ${ }^{1}$, Shaila Ghanekar', Jake Sokol ${ }^{1}$, John H. Zhang ${ }^{2}$ and Cesar V. Borlongan ${ }^{\text {* }}$
}

\begin{abstract}
Here, we compiled ten papers detailing recent strides in the field of stem cell therapy, which address critical issues relevant to pursuing this experimental treatment for clinical applications in brain disorders. Topics include efficacy, safety, and mechanism of action underlying cell therapy, emerging technologies and combination pharmacotherapies with cell transplantation directed at improving the functional outcomes, and evaluation of key translational components of advancing novel therapeutics to the clinic, including the need for vis-à-vis comparisons with the gold standard of treatment post-injury (i.e., physical rehabilitation). A bioethics paper is also incorporated here to further appreciate the current status of cell therapy in the community setting. The overall goal of this special volume is to provoke a meaningful assessment of the lessons we have learned in recent years and to use such knowledge to carefully translate safe and effective applications of cell therapy, and its mechanism of action for the treatment of neurological disorders.
\end{abstract}

Keywords: Brain disorders, Cell transplantation, Safety, Efficacy, Mechanism, Clinical trials, Bioethics, Biomaterials

\section{Background}

Stem cell therapy has emerged as a promising treatment for numerous neurological disorders. One such application has been recognized in stroke, a debilitating health burden that affects hundreds of thousands of individuals worldwide. Many patients would greatly benefit from the development of novel treatments for stroke with wider therapeutic windows than the current limited treatment, tPA (tissue plasminogen activator).

Several key clinical trials have helped shape the field of stem cell therapy, including the intracerebral transplantation of fetal cells and neuroteratocarcinoma-derived neural progentior cells (NT2N) cells in Parkinson's disease and stroke patients, respectively. Recent studies have explored the peripheral transplantation of stem cells for acute stroke patients, paving the way for minimally invasive cell therapy clinical studies.

\footnotetext{
* Correspondence: cborlong@health.usf.edu

${ }^{1}$ Center of Excellence for Aging \& Brain Repair, Department of Neurosurgery and Brain Repair, University of South Florida College of Medicine, 12901

Bruce B. Downs Blvd, Tampa, Florida 33612, USA

Full list of author information is available at the end of the article
}

The ten papers compiled in this special volume were selected from the recently concluded 2016 American Society for Neural Therapy and Repair (ASNTR) annual meeting, addressing various sources and profiles of stem cells and their therapeutic applications, cell death mechanisms that stand as key pathological bases underlying the stroke and therapeutic targets, and the prevailing bioethical concerns associated with cell-based treatments.

\section{Promising stem cells for cell therapy in neurological disorders}

Bone marrow-derived stem cells

Stroke is not only a prevalent disease, affecting hundreds of thousands throughout the United States, but its debilitating effects are rivaled by few. But despite the mortality and morbidity associated with stroke, available treatment options are limited. The standard treatment of acute ischemic stroke, tPA, is confined to a relatively minute subgroup of stroke patients due to its narrow therapeutic window of roughly $4.5 \mathrm{~h}$ post-stroke onset.

In the paper written by Vivian A. Guedes and coworkers of the Department of Neurosurgery and Brain Repair at the University of South Florida, USA, they 
explore stem cell therapy as a promising therapeutic alternative for stroke, specifically discussing different types of stem cells with potential for clinical use. Along with other factors such as route of administration, cell dose, and the sex of the donor/recipient, the type of stem cells likely influences the outcome of cell transplantation procedures. Dr. Guedes and colleagues first discuss embryonic stem cells (ES), then go on to explain the potential of bone marrow derived stem cells (BMDSCs), hematopoietic stem cells, mesenchymal stromal cells, endothelial progenitor cells (EPC), neural stem cells, and induced pluripotent stem cells. Although having diverse applications and positive experimental results, research on ES cells is hindered by ethical concerns. Still, the authors refer to substantial progress that has been made in ES cell research. Both endogenous and exogenous ES cells have been shown to have positive effects on the functional recovery of animals submitted to ischemic injuries. They also identify BMDSCs as an effective graft source for stroke, alluding to their ability to differentiate into neurons in vitro and secret growth factors involved in neuronal survival. Of the BMDSCs, the article elaborates primarily on EPCs, circling in peripheral blood capable of repairing damaged endothelium in the brain. Recent experiments have revealed peripheral injection of EPCs reduce infarct size in diabetic mice submitted to middle cerebral artery occlusion (MCAO), helping restore the microvasculature of the blood brain barrier [1]. Guedes and colleagues also examine the possible therapeutic advantage of co-transplantation, acknowledging its beneficial effects towards cell survival. Recent trials show the delivery of neural stem cells combined with adipose-derived stem cells increased neural stem cell survival [2]. Overall, the article skillfully examines the potential of various stem cells, providing valuable background for future discovery.

\section{MSC therapy for stroke}

As noted above, most stroke patients would greatly benefit from the development of promising treatments with wider therapeutic windows than the only approved treatment tPA. Dr. Jonathon D. Anderson and colleagues from the Department of Internal Medicine, University of California Davis, USA, use their review paper "Mesenchymal Stem Cell-based Therapy for Ischemic Stroke" to explore the use of mesenchymal stem cells (MSC) as a promising treatment for ischemic stroke.

MSCs are adult multipotent cells capable of differentiation toward various cell lineages. While MSCs themselves activate endogenous cellular repair programs, Dr. Anderson and colleagues have provided evidence through proteomic analysis that exosomes mediate much of MSCs regenerative capabilities with prosurvival and angiogenic proteins [3]. Recent studies by this research group also alluded to the potential of stem cells for stroke and other neurological disorders. For example, the potential of MSCs is discussed as a delivery platform for bioactive factors such as brainderived neurotrophic factors, BDNF [4] and as a foundation to enhance treatment of neurologic diseases, specifically Huntington's disease [5, 6]. The present review article and the authors' recent studies also demonstrate how the transplanted MSCs successfully migrate to the area of infraction and induce angiogenesis, neurogenesis, and neurite outgrowth through neuroprotective factors. Despite this evidence, the actual mechanism underlying MSCs therapeutic response upon injection is poorly understood. Further detailed studies exploring these effects would be greatly beneficial as MSCs continue to represent a promising treatment for ischemic stroke.

\section{MultiStem cell applications}

Dr. David Hess and colleagues discuss the lab-to-clinic application of stem cell therapy for ischemic stroke, specifically the use of MultiStem cells. The authors briefly emphasize intravenous delivery as a feasible method of administration of stem cells as it is available in the community hospital setting and provides sufficient cells throughout the body to contribute to treatment. The authors then go on to highlight MultiStem cells, a unique population of adherent bone marrow-derived cells, originally referred to as multipotent adult progenitor cells or MAPCs. This cell therapy product was developed based on Dr. Catherine Verfaillie's isolation procedure of MAPCs, multipotent cells capable of differentiating into cells from all three germ layers. Several preclinical studies investigating these cells' administration offer insights into the mechanisms underlying its efficacy, including immunomodulatory and regenerative properties $[7,8]$. In this review article, Dr. Hess and colleagues highlight the advantages of MultiStem cells compared to other stem cell alternatives, advancing them as a promising treatment of ischemic stroke. These cells have the potential to be manufactured in large scale and in uniform clinical doses, making them more easily distributed and available not only to academic medical centers but also community hospitals. Additionally, other recent studies demonstrated how MultiStem cells derived from either rats or humans exhibited comparable levels of functional recovery without the use of immunosuppression [9]. The authors also further discuss the potential of MultiStem cell therapy because of its wider time window and no requirement for tissue matching. The authors, based on Dr. David Hess' pioneering clinical trial on Multistem, effectively provide insight on the potential of MultiStem cells as a promising treatment for stroke, allowing more patients to benefit from such a debilitating burden.

\section{Amniotic fluid stem cell potential}

Although amniotic fluid stem cells (AFSC) display many clear clinical advantages over amniotic membrane stem 
cells (AMSC), the majority of research continues to focus on AMSC. In the present paper "Translating amniotic fluid-derived stem cells for transplantation in stroke," Jake Sokol and collaborators, they presented the scientific basis that warrants the need for exploring AFSC's full therapeutic potential, especially in the treatment of stroke.

The authors discuss recent experiments by Antonucci et al. [10] to demonstrate the pluripotency of AFSCs and their ability to differentiate into all three germ layers and many different cell types, such as adipocytes, osteocytes, chondrocytes, and neuronal cells. After justifying the cells as having the properties of stem cells, they then explain the potential of AFSCs in the treatment of stroke. Of note, AFSC administration aids in the regeneration of neural tissue of stroke rats and attenuates deficits in memory and learning, providing evidence of the potential application of AFSCs in treatment of stroke for humans.

Next, the authors then compare the advantages of disadvantages of AFSCs and the more researched AMSCs, detailing their mode of harvesting, time-frame of administration, and ease of amplification and differentiation. AFSCs have one clear advantage over AMSCs in their distinct collection procedures; AFSCs can be harvested while the mother is still pregnant. This procedure, known as amniocentesis, allows the cells more time to be collected and amplified. So, when the child is delivered, defects can be more easily treated using the child's own stem cells. However, the authors explain how this can sometimes be seen as a disadvantage, as amniocentesis risks harm to both the mother and the child. On the other hand, AMSCs can only be collected after birth, so no risk is posed to either party. But although there may be no risk from the cell harvesting, the cells cannot be immediately administered to the newborn child, as the cells must have time to differentiate. Accordingly, if a child must be treated in a critical therapeutic window following birth, the more viable source of stem cells would be AFSCs. Another disadvantage of using AFSCs is that there origin is not well studied, so it is difficult to isolate and confirm the stem cells' lineage.

Despite the limitations of AFSCs, Sokol and colleagues advance their thesis by discussing the regenerative potential of AFSCs, describing their ability to enhance the therapeutic outcome of allograft acceptance. The authors refer to several key studies that have shown how the immunomodulatory properties of AFMSCs help to promote allograft acceptance in the long run. Overall, the article effectively argues and communicates to the reader the potential of AFSCs and the importance of future research to continue discovering their many applications for brain disorders beyond stroke.

\section{Cell death mechanisms mediating neurological disorders as therapeutic targets for cell therapy Mitochondrial regulation of calcium uptake}

Calcium overload plays a very important role in cell death after cerebral ischemia. The uptake of calcium is known to be regulated by the mitochondrial calcium uniporter (MCU) and a rise in calcium signals an increased demand for cellular energy, hastening the path towards mitochondrial injury and death. Dr. Kun Zhang of the Department of Neurology at the Second Hospital of Hebei Medical University, China, and colleagues at Georgetown University and University of Washington assert the role of mitochondrial calcium uptake 1(MICU1) as a key activator of MCU at high calcium levels and its relevance in cerebral ischemia in their paper on "The expression of MICU1 after Cerebral Ischemia in MCAO Rat Model." Previously, it was shown that an influx of calcium, along with other factors such as glutamate release, is responsible for cell death [11]. The study emphasizes the fact that an accumulation of mitochondrial calcium causes the opening of a pore in the inner mitochondrial membrane, a lowering of the membrane potential, and the blockade of the production of ATP [11]. The present authors also recently demonstrated the effects of calcium overload and mitochondrial dysfunction in triggering glutamate-induced neuronal death. They found that an inhibition of MCU prevented glutamate- induced cell death by limiting the amount of calcium that could enter the mitochondria, preventing the opening of the mitochondrial permeability transition pore by glutamate, and avoiding the possibility of a calcium overload [12]. Although it has been found several times that MCU is the main vehicle for calcium uptake, the regulator of MCU is yet to be fully determined.

Dr. Zhang and colleagues' present paper highlights the role of MICU1 as a key regulator of MCU, and therefore mitochondrial calcium uptake. In the experiment, they tested the expression of MICU1 at both the protein and mRNA level at various time periods after permanent middle cerebral artery occlusion in Sprague Dawley rats. The results displayed a significant increase in MICU1 expression at both the protein and mRNA level in the 6-12 h period after ischemia. Dr. Zhang and associates go on to address the significance of their findings, most notably the connection between an increase in MICU1 levels and a previously discovered increase in calcium levels in the $6-12 \mathrm{~h}$ period. This connection provides significant evidence of MICU1's role in calcium homeostasis. Considering calcium's role in cell death in the ischemic brain, Dr. Zhang and collaborators' findings represent a novel discovery. Further research into MICU1 could allow for the possibility of limiting cell death after ischemia through the manipulation of MICU1 levels in the brain. Coupled with stem cell therapy, these 
findings and further investigations could help to decrease the incidence of stroke across the globe.

\section{Cellular link between ischemic stroke and cardiac failure} Cerebrovascular and cardiovascular diseases are the two leading causes of death in the world. Currently, both illnesses are treated separately, however, evidence has surfaced indicating a close pathological link between the two. In their paper on "Pathological links between stroke and cardiac arrest" Shaila Ghanekar of the Department of Neurosurgery and Brain Repair at the University of South Florida, USA, and her colleagues highlight the clinical findings that indicate a cellular link between ischemic stroke and cardiac failure and assert the necessity to develop novel treatments that assess both diseases. Previous studies have affirmed the connection between cerebral ischemia and heart failure. In a study conducted in 2006, the researchers discovered that the risk of death due to cardiac failure doubled after the incidence of ischemic stroke [13]. However, the exact pathway that connects the two diseases has yet to be determined.

Accumulating evidence advances the importance of determining the direct cell death pathway between ischemic stroke and cardiac failure in order to further the development of innovative treatments that address both diseases [14, 15]. Ghanekar and her collaborators first highlight the shared risk factors of both diseases, including hypertension and diabetes, as well as asserting that after a stroke, high levels of cardiac enzymes exist in the body, indicating the presence of stressed or dying cardiac cells. The authors go on to affirm the role of the insular cortex in facilitating cardiac myocyte death, advocating for further research on the connection between the insular cortex and the heart. In an in vitro study, an oxygen-glucose deprivation model using primary rat neuronal cells (PRNCs) was utilized to determine the cellular alterations in rat cardiac myocytes (RCMs) after cerebral ischemia. The results revealed that the PRNCs caused toxic effects on the RCMs. In the invivo study, transient middle MCAO was induced and immunhistochemical analyses were conducted on both the brains and hearts of the rats. The study revealed death of RCMs through the conference of cell death signals from the ischemic brain to the heart. With the results gained from this study [14], Ghanekar and her colleagues address these findings and their clinical implications in this review paper. Considering the insular cortex is responsible for controlling heart rhythm, they assert that stroke in this region could result in arrhythmias or even sudden death. The in vitro study also identified autophagy-related cell death signals could be responsible for cardiac failure following stroke [14], indicating that while stroke may initiate in one area, the damage can spread to the rest of the brain and possibly the heart. The presence of elevated levels of brain natriuretic peptide (BNP) and N-terminal-pro-brain natriuretic peptide (NT-proBNP) following ischemic stroke suggests the role of the kidney in stroke pathology. The present review paper supports the concept of direct cellular pathways between the ischemic brain and cardiac failure, which could pave the way to future investigations on the connections between these two diseases. Together with further stem cell research, the development of potential treatments incorporating the concept of brain-heart pathological link could effectively combat both cerebral ischemia and cardiac complications.

\section{Improving the efficacy of stem cells Biomaterials as adjunct treatments for stem cell transplantation}

One of the main symptoms of neurological conditions, like stroke, is the loss of functional neural tissue. This is currently combatted by physical therapy and regenerative approaches, such as stem cell therapy, however, both have presented with limited success. A proponent of the use of hydrogels and other biomaterials in neural tissue regeneration, Dr. Harman Ghuman and Dr. Michael Modo of the Department of Bioengineering at the University of Pittsburgh, USA assert the overarching benefits of using biomaterials in their paper on "Biomaterial applications in neural therapy and repair." The authors have conducted previous studies that prove the efficacy of hydrogels in the treatment of stroke. In an earlier study, they addressed the particular structure-function relationship of biomaterials and the necessity for testing and confirming the existence of an injectable form [16]. In a recent study, they tested the use of an acellular extracellular matrix in encouraging the infiltration of host brain cells and replacing necrotic debris. They found that certain concentrations of the hydrogel were capable of promoting an endogenous repair response that could be essential for post-stroke neural repair [17].

Drs. Ghuman and Modo's present review paper highlights the prevalence of biomaterials considering the seemingly irreversible damage caused by stroke. They assert the role of hydrogels as possible scaffolds to promote interactions between the host and transplanted tissues, occupy the tissue cavity, promote the attachment and engraftment of transplanted cells, and control sitespecific delivery of drugs and growth factors. The authors address the fact that the post-stroke microenvironment is not at all suitable for implantation of stem cells, making the presence of a biomaterial necessary to stabilize the environment. They also go on to describe the importance of select characteristics, such as biocompatibility and biodegradability, in order to ensure that the injection of a host-compatible biomaterial will 
minimize an aggravated response from immune cells. They affirm the use of both natural and synthetic polymers, collagen and poly(DL-lactic-co-glycolic acid) (PLGA) accordingly, and their various advantages and disadvantages. In addition, they assert the importance of biomaterials in the delivery of exogenous cells and bioactive molecules to the damaged region, preventing the need for multiple injections and excess damage to the peri-infarct brain tissue. Furthermore, Drs. Ghuman and Modo highlight the potential of endogenous neurogenesis, assisted by the presence of a biomaterial that would direct neural progenitor cells directly to the site. The review paper advances the importance and efficacy of biomaterials as an adjunct treatment to cell-based stroke therapy. A careful analysis of future lab-to-clinic translational obstacles will allow realization of the implementation of biomaterials in stroke patients. The authors provide valuable insights into the possibilities of biomaterial use and its future role as an effective stroke therapy.

\section{Improving endogenous neurorepair through pharmacological therapy}

To date, drug therapy for stroke is severely limited due to unpredictable onset of the disease. As highlighted above, the standard treatment of acute ischemic stroke, tPA, is confined to a relatively minute subgroup of stroke patients due to its narrow therapeutic window of roughly $4.5 \mathrm{~h}$ post-stroke onset. However, several researched chemicals, peptides or trophic factors, have been discovered that may improve behavioral recovery when administered even beyond this short window post-stroke. Among the many biological mechanisms, targeting endogenous repair of the neuroprogenitor cells (NPC) in the subventricular zone (SVZ) shows promise for these newly discovered factors, which may enable longer treatment windows for stroke patients.

After stroke, de novo neurogenesis has been found in the SVZ of adult mammalian brain, but most of the newly formed brain cells die shortly after insult. However, recent studies have determined that pharmacological manipulation may improve the survival of endogenous NPCs and neural regeneration in stroke rats. In the article written by Dr. Kuo-Jen Wu and colleagues of the National Health Research Institutes in Miaoli, Taiwan, they discuss several candidate chemicals recently developed to modulate survival proliferation, migration, or differentiation of NPCs in SVZ. The authors first discuss Pifithrin- $\alpha$ (PFTa), a p53 inhibitor, and then take note of its tendency to extend the survival of endogenous NPCs [18]. The authors also highlight several trophic factors, bone morphogenetic protein 7 (BMP7) and Brain-derived neurotrophic factor (BDNF), that have been shown to enhance neuroreparative activity post-stroke. For example, the article refers to a key study in which post-treatment with BMP7 enhanced recovery of sensorimotor function in the impaired limbs, decreased body asymmetry, and increased locomotor activity [19]. Also, a recent study demonstrated how administration of BDNF enhanced migration of NPCs from SVZ toward the olfactory bulbs of the animal [20-22]. The present paper also emphasizes the importance of preventative measures in the pre-stroke period, particularly discussing the peptide cocaine and amphetamine-regulated transcript (CART) and its potential to reduce cerebral infarction in mice. Overall, the article effectively examines the potential of various stroke drug treatments and preemptive measures with potential to revolutionize modern stroke treatment.

\section{The future of stem cell therapy}

Stem cell therapy: current status and future implications

In their manuscript "Translational lab-to-clinic hurdles in stem cell therapy," Sydney Corey and her colleagues of the Department of Neurosurgery and Brain Repair at the University of South Florida College of Medicine, USA, explore various topics and challenges regarding stem cells as well as their future implications. Several key clinical trials have helped shape the field of stem cell therapy, including the clinical experts who pioneered the Parkinson's clinical trials [23, 24], as well as the recent exploration of peripheral transplantation in acute stroke patients [25]. In this article, Corey and her team discuss the combination treatments of stem cell therapy and rehabilitation therapy as a key challenge to optimize clinical benefits for stroke patients. They recognize the severe disconnect between the laboratory and the clinical settings which have overlooked the use of rehabilitation therapy despite its proven benefits in endogenous cell therapy. They go on to review various sources of stem cells, deeming adult stem cells the "most attractive" currently due to the previous federal moratorium on National Institutes of Health (NIH) funds towards embryonic stem cell research. Additionally, the authors discusses both peripheral and intracerebral approaches as promising administration routes for transplantation, while also recognizing that repeated/booster transplants may be required. As for the most relevant animal models for stem cell research, small rodents are identified as being instrumental in studies, yet limitations exist due to the white matter that is not representative of the human brain. Corey and her collaborators further evaluate how functional recovery can be measured in animal models, pointing out that while motor function remains the most cost-effective and popular method, cognitive function must also be assessed to capture the full range of therapeutic potential of stem cell therapy. Updates on the ongoing trials of cell therapy will provide valuable information for stem cells and their future applications for the common good. 


\section{Bridging the gap between the public and scientific community}

The bioethics of stem cell-based therapy continues to be a topic of controversy due to the disconnect between the scientific community and the general public. The misperception and lack of awareness has led to negative media coverage and unfavorable public opinion, hindering the progress of scientific study. In the review paper "Media coverage and public awareness on bioethics perception of emerging biomedical therapies", Dr. Sandra Acosta and her collaborators of the Department of Neurosurgery and Brain Repair at the University of South Florida College of Medicine, USA, present an overview of the ethical issues surrounding stem cell-based therapies and advances the concept of establishing a consortium to bring forth guidance and understanding by identifying and analyzing legal, social, and moral issues.

Dr. Acosta and colleagues have provided preclinical evidence of safety and efficacy of stem cell therapy in traumatic brain injury [26, 27] and in stroke [28, 29]. In this article, they highlight the bioethical issues that have accompanied this cell therapy over the last two decades and raised public concern, including safety measures, commercialization, and confidentiality of donors. The authors' emphasis is on the heavy influence of the media that has exploited the absence of successful communication between the public and the scientific community. Consequently, developing technologies that have the capability to revolutionize healthcare are stifled by public fear and misperception. Dr. Acosta and colleagues thus propose the establishment of a consortium to bridge the gap between the scientific community and the general public by addressing bioethical issues and public opinion. In order to establish a baseline public conclusion about stem cell research, the authors explore the concept of a conglomerate with focus subgroups to develop training programs on the ethics of emerging technologies. While the review paper advances this concept, similar regulatory panels such as those convened by the NIH to govern stem cell research exist. Accordingly, compilations of guidelines arising from consortia within the US and around the world may provide better consensus among stakeholders on the bioethics of stem cells. Nonetheless, Dr. Acosta and her team provide valuable insight on the importance of ensuring that public communication does not inhibit the progression of stem cell research.

\section{Conclusion}

In summary, these ten selected articles from the recent ASTNR meeting highlight the potential applications for stem cell therapy in neurological disorders, detailing the laboratory evidence on the safety, efficacy, and mechanism of action of these transplantable cells, altogether forming the basis for the clinical trials [30-33]. Given the prevalence of stroke, many patients would greatly benefit from novel treatments that capitalize on the promising therapeutic benefits of stem cells. Numerous studies referenced throughout the articles have indicated the potential capability of stem cell therapy in regenerating the diseased brain. We are gaining more insights into the cellular death pathways mediating stroke [34-39], and in parallel exploiting these novel diseaserelated mechanisms as therapeutic targets for stem cells to exert their functional benefits. Yet, these developing technologies face obstacles, such as bioethical concerns and misrepresentations, as well as exploitations by the unscrupulous media and business sector, preventing them from reaching full beneficial capacity. Accordingly, a common theme resonating throughout these papers is a push to increase translational research of cell-based therapeutics for clinical applications that will allow scientifically sound assessments of cell therapy, delineating hype from hope [40-48]. Forthcoming updates on the ongoing clinical trials of cell therapy will provide valuable information on which to build the future of stem cell research and therapeutic applications.

\section{Abbreviations \\ AFSC: amniotic fluid stem cells; AMSC: amniotic membrane stem cells; ASNTR: American Society for Neural Therapy and Repair; BDNF: brain-derived neurotrophic factors; BM: bone marrow; BMDSCs: bone marrow-derived stem cells; BMP7: bone morphogenetic protein 7; BNP: brain natriuretic peptide; CART: cocaine and amphetamine-regulated transcript; EPC: endothelial progenitor cells; ES: embryonic stem cells; MAPCs: multipotent adult progenitor cells; MCAO: middle cerebral artery occlusion; MICU1: mitochondrial calcium uptake 1; MSC: mesenchymal stem cells; NIH: National Institutes of Health; NPC: neuroprogenitor cells; NT2N: neuroteratocarcinoma-derived neural progentior cells; NT-proBNP: N-terminal-pro-brain natriuretic peptide; PFTa: Pifithrin-a; PLGA: poly(DL-lactic-co-glycolic acid); PRNCs: primary rat neuronal cells; RCMs: rat cardiac myocytes; SVZ: subventricular zone; tPA: tissue plasminogen activator}

\section{Acknowledgement}

The authors thank the participants of 2016 ASNTR who contributed to this Chinese of Neurosurgical Journal special volume.

\section{Funding}

CVB is funded by NIH R01NS071956, NIH R01 NS090962, NIH R21NS089851, NIH R21 NS094087, DOD W81XWH-11-1-0634, and VA Merit Review 101 BX001407.

\section{Availability of data and materials}

This paper is a review article. Referred literature in this paper has been listed in the references part. The datasets supporting the conclusions of this article are available online by searching the PubMed. Some original points in this article come from the laboratory practice in our research centers and the authors' experiences.

Authors' contributions

The authors contributed equally to the conceptualization and write-up of this manuscript. All authors read and approved the final manuscript.

Competing interests

The authors declare no competing interests.

Consent for publication

Not applicable. 


\section{Ethics approval and consent to participate}

Not applicable.

\section{Author details}

${ }^{1}$ Center of Excellence for Aging \& Brain Repair, Department of Neurosurgery and Brain Repair, University of South Florida College of Medicine, 12901 Bruce B. Downs Blvd, Tampa, Florida 33612, USA. ²Departments of Anesthesiology, Neurosurgery, Neurology and Physiology, Loma Linda University Medical Center, 11234 Anderson St, Loma Linda, CA 92354, USA.

\section{Received: 14 July 2016 Accepted: 8 December 2016}

Published online: 16 January 2017

\section{References}

1. Borlongan CV, Glover LE, Tajiri N, Kaneko Y, Freeman TB. The great migration of bone marrow-derived stem cells toward the ischemic brain: Therapeutic implications for stroke and other neurological disorders. Prog Neurobiol. 2011;95:213-28

2. Oh JS, Kim KN, An SS, Pennant WA, Kim HJ, Gwak SJ, Yoon do H, Lim MH, Choi $\mathrm{BH}, \mathrm{Ha}$ Y. Cotransplantation of mouse neural stem cells (mNSCs) with adipose tissue-derived mesenchymal stem cells improves mNSC survival in a rat spinal cord injury model. Cell Transplant. 2011;20:837-49.

3. Anderson JD, Johansson HJ, Graham CS, et al. Comprehensive Proteomic Analysis of Mesenchymal Stem Cell Exosomes Reveals Modulation of Angiogenesis via NFkB Signaling. Stem Cells. 2016;34:601-13.

4. Deng P, Anderson JD, Yu AS, Annett G, Fink KD, Nolta JA. Engineered BDNF producing cells as a potential treatment for neurologic disease. Expert Opin Biol Ther. 2016;21:1-9.

5. Nolta JA. "Next-generation" mesenchymal stem or stromal cells for the in vivo delivery of bioactive factors: progressing toward the clinic. Transfusion. 2016:56:15S-7S.

6. Fink KD, Deng P, Gutierrez J, Anderson JS, Torrest A, Komarla A, Kalomoiris S, Cary W, Anderson JD, Gruenloh W, Duffy A, Tempkin T, Annett G, Wheelock V, Segal DJ, Nolta JA. Allele-Specific Reduction of the Mutant Huntingtin Allele Using Transcription Activator-Like Effectors in Human Huntington's Disease Fibroblasts. Cell Transplant. 2016;25:677-86.

7. Van Bokkelen G. Company profile: Athersys. Regen Med. 2011;6:39-43.

8. Yasuhara T, Hara K, Maki M, et al. Intravenous grafts recapitulate the neurorestoration afforded by intracerebrally delivered multipotent adult progenitor cells in neonatal hypoxic-ischemic rats. J Cereb Blood Flow Metab. 2008:28:1804-10.

9. Mays RW, Borlongan CV, Yasuhara T, Hara K, Maki M, Carroll JE, Deans RT, Hess DC. Development of an allogeneic adherent stem cell therapy for treatment of ischemic stroke. J Exp Stroke Transl Med. 2010;3:34-46.

10. Antonucci I, Di Pietro R, Alfonsi M, et al. Human second trimester amniotic fluid cells are able to create embryoid body-like structures in vitro and to show typical expression profiles of embryonic and primordial germ cells. Cell Transplant. 2014;23:1501-15.

11. Kristián T, Siesjö Bo K, et al. Calcium in Ischemic Cell Death. Stroke. 1998;29: 705-718

12. Liu X, Xu S, Wang P, Wang W. Transient mitochondrial permeability transition mediates excitotoxicity in glutamate-sensitive NSC34D motor neuron-like cells. Exp Neurol. 2015;271:122-30.

13. Dhamoon MS, Sciacca RR, Rundek T, et al. Recurrent stroke and cardiac risks after first ischemic stroke: the Northern Manhattan Study. Neurology. 2006;66:641-6

14. Ishikawa H, Tajiri N, Shinozuka K, Vasconcellos J, Kaneko Y, Lee HJ, Mimura O, Dezawa M, Kim SU, Borlongan CV. Vasculogenesis in experimental stroke after human cerebral endothelial cell transplantation. Stroke. 2013:44:3473-81.

15. Ishikawa H, Tajiri N, Vasconcellos J, Kaneko Y, Mimura O, Dezawa M, Borlongan CV. Ischemic stroke brain sends indirect cell death signals to the heart. Stroke. 2013;44:3175-82.

16. Massensini AR, Ghuman H, Saldin LT, Medberry CJ, Keane TJ, Nicholls FJ, et al. Concentration-dependent rheological properties of ECM hydrogel for intracerebral delivery to a stroke cavity. Acta Biomater. 2015;27:116-30

17. Ghuman H, Massensini AR, Donnelly J, Kim SM, Medberry CJ, Badylak SF, et al. ECM hydrogel for the treatment of stroke: Characterization of the host cell infiltrate. Biomaterials. 2016;91:166-81.

18. Luo Y, Kuo CC, Shen H, Chou J, Greig NH, Hoffer BJ, Wang Y. Delayed treatment with a p53 inhibitor enhances recovery in stroke brain. Ann Neurol. 2009;65:520-30.
19. Chang CF, Lin SZ, Chiang YH, Morales M, Chou J, Lein P, Chen HL, Hoffer BJ, Wang Y. Intravenous admininstration of Bone morphogenetic protein-7 after ischemia improves motor function in stroke rats. Stroke. 2003;34:558-64.

20. Bagley JA, Belluscio L. Dynamic imaging reveals that brain-derived neurotrophic factor can independently regulate motility and direction of neuroblasts within the rostral migratory stream. Neuroscience. 2010;169:1449-61.

21. Bath KG, Akins MR, Lee FS. BDNF control of adult SVZ neurogenesis. Dev Psychobiol. 2012;54:578-89.

22. Chiaramello S, Dalmasso G, Bezin L, Marcel D, Jourdan F, Peretto P, Fasolo A De MS. BDNF/ TrkB interaction regulates migration of SVZ precursor cells via PI3-K and MAP-K signalling pathways. Eur J Neurosci. 2007;26:1780-90.

23. Kordower JH, Freeman TB, Vingerhoets FJ, et al. Neuropathological evidence of graft survival and striatal reinnervation after the transplantation of fetal mesencephalic tissue in a patient with Parkinson's disease. N Engl J Med. 1995:332(17):1118-24.

24. Lindvall O, Backlund EO, Farde L, et al. Transplantation in Parkinson's disease: two cases of adrenal medullary grafts to the putamen. Ann Neurol. 1987;22(4):457-68

25. Savitz SI, Cramer SC, Wechsler L. Stem cells as an emerging paradigm in stroke 3: enhancing the development of clinical trials. Stroke. 2014;45:634-9.

26. de la Peña I, Sanberg PR, Acosta S, Lin SZ, Borlongan CV. Umbilical cord blood cell and granulocyte-colony stimulating factor: combination therapy for traumatic brain injury. Regen Med. 2014:9:409-12.

27. De La Peña I, Sanberg PR, Acosta S, Lin SZ, Borlongan CV. G-CSF as an adjunctive therapy with umbilical cord blood cell transplantation for traumatic brain injury. Cell Transplant. 2015;24:447-57.

28. Acosta SA, Tajiri N, Hoover J, Kaneko Y, Borlongan CV. Intravenous Bone Marrow Stem Cell Grafts Preferentially Migrate to Spleen and Abrogate Chronic Inflammation in Stroke. Stroke. 2015:46:2616-27.

29. Tajiri N, Acosta S, Portillo-Gonzales GS, Aguirre D, Reyes S, Lozano D, Pabon M, Dela Peña I, Ji X, Yasuhara T, Date I, Solomita MA, Antonucci I, Stuppia L, Kaneko Y, Borlongan CV. Therapeutic outcomes of transplantation of amniotic fluid-derived stem cells in experimental ischemic stroke. Front Cell Neurosci. 2014:8:227.

30. Napoli E, Borlongan CV. Recent advances in stem cell-based therapeutics for stroke. Transl Stroke Res. 2016 Aug 12. [Epub ahead of print]

31. Borlongan CV. Age of PISCES: stem-cell clinical trials in stroke. Lancet. 2016; 388:736-8.

32. Steinberg GK, Kondziolka D, Wechsler LR, Lunsford LD, Coburn ML, Billigen JB, Kim AS, Johnson JN, Bates D, King B, Case C, McGrogan M, Yankee EW, Schwartz NE. Clinical outcomes of transplanted modified bone marrowderived mesenchymal stem cells in stroke: A phase 1/2a study. Stroke. 2016; 47:1817-24.

33. Kalladka D, Sinden J, Pollock K, Haig C, McLean J, Smith W, McConnachie A, Santosh C, Bath PM, Dunn L, Muir KW. Human neural stem cells in patients with chronic ischaemic stroke (PISCES): a phase 1, first-in-man study. Lancet. 2016:388:787-96.

34. Liska MG, Crowley MG, Nguyen H, Borlongan CV. Implication of biobridge concept in stem cell therapy for ischemic stroke. J Neurosurg Sci. 2016 Jul 12 [Epub ahead of print]

35. Nguyen H, Aum D, Mashkouri S, Rao G, Vega Gonzales-Portillo JD, Reyes S, Borlongan CV. Growth factor therapy sequesters inflammation in affording neuroprotection in cerebrovascular diseases. Expert Rev Neurother. 2016;16:915-26

36. Borlongan $\mathrm{CV}$, Jolkkonen J, Detante $\mathrm{O}$. The future of stem cell therapy for stroke rehabilitation. Future Neurol. 2015;10:313-9.

37. Tajiri N, Lee JY, Acosta S, Sanberg PR, Borlongan CV. Breaking the blood-brain barrier with mannitol to aid stem cell therapeutics in the chronic stroke brain. Cell Transplant. 2016;25:1453-60.

38. Gonzales-Portillo C, Ishikawa H, Shinozuka K, Tajiri N, Kaneko Y, Borlongan CV. Stroke and cardiac cell death: Two peas in a pod. Clin Neurol Neurosurg. 2016;142:145-7.

39. Id P, Borlongan CV. Translating G-CSF as an adjunct therapy to stem cell transplantation for stroke. Transl Stroke Res. 2015:6:421-9.

40. Borlongan CV, Chopp M, Steinberg GK, Bliss TM, Li Y, Lu M, Hess DC, Kondziolka D. Potential of stem/progenitor cells in treating stroke: the missing steps in translating cell therapy from laboratory to clinic. Regen Med. 2008:3:249-50

41. Park DH, Borlongan CV, Willing AE, Eve DJ, Cruz LE, Sanberg CD, Chung YG, Sanberg PR. Human umbilical cord blood cell grafts for brain ischemia. Cell Transplant. 2009;18:985-98. 
42. Nishino $\mathrm{H}$, Borlongan CV. Restoration of function by neural transplantation in the ischemic brain. Prog Brain Res. 2000;127:461-76.

43. Borlongan CV. Bone marrow stem cell mobilization in stroke: a 'bonehead' may be good after all! Leukemia. 2011;25:1674-86.

44. Newman MB, Misiuta I, Willing AE, Zigova T, Karl RC, Borlongan CV, Sanberg PR. Tumorigenicity issues of embryonic carcinoma-derived stem cells: relevance to surgical trials using NT2 and hNT neural cells. Stem Cells Dev. 2005;14:29-43.

45. Zigova T, Willing AE, Tedesco EM, Borlongan CV, Saporta S, Snable GL, Sanberg PR. Lithium chloride induces the expression of tyrosine hydroxylase in hNT neurons. Exp Neurol. 1999;157:251-8.

46. Hara K, Yasuhara T, Maki M, Matsukawa N, Masuda T, Yu SJ, Ali M, Yu G, Xu L, Kim SU, Hess DC, Borlongan CV. Neural progenitor NT2N cell lines from teratocarcinoma for transplantation therapy in stroke. Prog Neurobiol. 2008; 85:318-34.

47. Diamandis T, Borlongan CV. One, two, three steps toward cell therapy for stroke. Stroke. 2015:46:588-91.

48. Elias M, Hoover J, Nguyen H, Reyes S, Lawton C, Borlongan CV. Stroke therapy: the potential of amniotic fluid-derived stem cells. Future Neurol. 2015;10:321-6.

Submit your next manuscript to BioMed Central and we will help you at every step:

- We accept pre-submission inquiries

- Our selector tool helps you to find the most relevant journal

- We provide round the clock customer support

- Convenient online submission

- Thorough peer review

- Inclusion in PubMed and all major indexing services

- Maximum visibility for your research

Submit your manuscript at www.biomedcentral.com/submit
() Biomed Central 